Aus der Chirurgischen Privatheilanstalt Dr. Ha as, München.

\title{
Über Hyperorchidie.
}

\author{
Von Dr. Alfred Haas, München.
}

(Mit I Abbildung.)

Ko che $\mathbf{r}$ hat eine $\ddot{~}$ berzahl der männlichen Keimdrüse nur beim echten Hermaphroditismus beobachtet. K ö n ig erklärt, daß es keinen mit Sicherheit beobachteten Fall gäbe, bei dem sich Täuschung durch Tumorenbildung, insbesondere durch umschriebene Hydrocelen und Zysten des Samenstranges ausschließen lasse. Im Handbuch der praktischen Chirurgie, in den Lehrbüchern der speziellen Chirurgie, so in dem von Hochenegg. und Payr, in dem neuen von Garrè und Borchardt ist unter den Mißbildungen der männlichen Genitalien nirgendwo von Mehr-Hodenbildung die Rede.

Nichtsdestoweniger gibt es in der Literatur mehrere, sicher beobachtete Fälle. Der älteste exakt beobachtete stammt von Le Dentu, der bei einer Sektion auf der rechten Seite zwei Hoden fand, die völlig dem linken glichen. Losse $n$ hat ein Präparat von Hyperorchidie beschrieben und mikroskopisch untersucht. Es handelt sich um den exstirpierten rechtsseitigen $\mathrm{Hy}$ drocelensack eines 28jährigen Mannes, bei dem klinisch neben dern normalen linken Hoden ein rechter nicht getastet werden konnte. In der $\mathrm{W}$ andung des Sackes, und zwar an der Grenze des oberen Drittels und am Grund fanden sich je ein kleiner rundlicher Tumor, die beide mikroskopisch als Hoden erkannt wurden; an beiden bestanden Nebenhoden, ein beide Nebenhoden verbindender, zuerst als Ductus deferens angesehener Strang erwies sicli mikroskopisch als aus Nebenhodenkanälchen bestehend, so daß die beiden Nebenhoden als Einheit aufgefaßt werden mußten. Von dem unteren Pol des tieferen Nebenhodenteiles ging dann e in Ductus deferens ab, der nur ein Lumen hatte. Beide Hoden zeigten gute Spermatozoenbildung. 
Pota r ca fand bei der Operation einer linksseitigen Hydrocele zwei taubeneigroße Hoden, beide kleiner als der rechte; der eine stand $2 \mathrm{~cm}$ höher als der andere, es fand sich keine Epididymis, aber zwei Samenstränge und zwei Samenleiter. Beide Hoden wurden bei der Operation belassen.

Ma r i o t t i stellte bei der Radikaloperation einer linksseitigen Leistenhernie neben einem normalen Hoden mit Samenstrang ein bohnengroßes Gebilde fest, zu dem ein zweiter linker Samenstrang hinzog. Dic histologische Untersuchung nach der Resektion ergab Hodengewebe.

De Franceschi operierte bei einem i 8 jährigen Mann eine linksseitige Leistenhernie und fand neben einem normal großen Hoden ein kleines, taubeneigroßes Gebilde mit nebenhodenähnlicher Ausbuchtung und einem federkieldicken, $3 \mathrm{~cm}$ langen Strang, der zum Samenstrang des Haupthodens hinzog. Leider fehlt eine mikroskopische Untersuchung dieses Falles.

$\mathrm{W}$ i d h a $1 \mathrm{~m}$ operierte eine linksseitige Leistenhernie mit mehrkammriger Hydrocele und fand in einer der Hydrocelenkammern zwei gleich große, hart nebeneinanderliegende Hoden, die durch einen breiten Nebenhoden an ihrer Rückfläche verbunden waren. Zwei getrennte Ductus deferentes gingen von letzterem ab, ihr getrennter Verlauf ließ sich im ganzen Samenstrang verfolgen.

Drenkhahn und Ayyer haben je einen Fall von Triorchismus beschrieben, die sie bei Musterungen beobachteten, der erstere bei einem deutschen Musketier, der letztere bei einem 19jährigen Südinder. Beide fanden die Mißbildung linkerseits und gründen ihre nicht autoptisch belegte Diagnose auf den Tastbefund, hauptsächlich auf die typische Hodensensation bei Druck.

Beide Fälle können nicht als beweiskräftig angesehen werden, weil die Täuschungsmöglichkeit beim rein palpatorischen Nachweis eine zu große ist, insbesondere genügt das spezifische Druckgefühl des Hodens nicht zur Diagnose. Hat doch schon Le D e ntu gezeigt, daß dieses Zeichen nicht pathognomonisch ist, da jede mit dem Plexus spermaticus zusammenhängende Neubildung bei Kompression eine der bei Hodendruck ausgelösten analoge Empfindung gibt. 
Es verbleiben also in der bisherigen Literatur 6 autoptisch belegte Fälle von Überhoden; histologisch untersucht sind nur Fall Lossen und Mariotti; von letzterem stehen mir leider nicht die genauen Details zur Verfügung, da mir nur das Referat zugänglich war.

An meiner Anstalt kam im Juli I 9 I 9 folgender Fall zur Beobachtung, dessen Krankengeschichte ich kurz wiedergebe:

F. M., 9 Jahre alter Knabe. Dic Eltern bringen ihn, weil ein beiderseitiger Leistenbruch besteht und wcil in seinem Hodensack keine Hoden sind. Der objektive Befund war folgender: Gut entwickelter Knabe von gesundem Aussehen. Innere Organe ohne krankhaften Befund. Scrotum klein, leer. In beide Leisten wölben sich beim Pressen kleine Hernien vor, Hoden in den Leistenkanälen nicht mit Sicherheit zu tasten. Penis dem Alter entsprechend mäßige Phimose. Operation am I8. VII. 1919. Leistenschnitt rechts. Freilegung des Leistenkanals durch Spaltung der Externusfascie. Es fand sich hier ein kleinhaselnußgroßer, kugeliger Hoden in kongenitalem Bruchsack. Der Bruchsack wird isoliert und abgetragen, der Hoden nach Dehnung des Samenstranggebildes ins Scrotum hinuntergezogen, das Gubernaculum mit einem Seidenfaden durchflochten und mit diesem der Hoden am Grund des Scrotums fixiert und gegen die Innenfläche des rechten Oberschenkels extendiert. Der Leistenkanal wird nach B as s in i-D reesmann geschlossen. Freilegung des linksseitigen Leistenkanals; in ihm findet sich zunächst ein über erbsengroßes Gebilde, das dem Hoden zu entsprechen scheint. Ein deutliches Vas deferens und mehrere Gefäße ziehen zu ihm hin. Nach Eröffnung des Peritonealtrichters kommt aber cin zweiter Hoden zum Vorschein, der in der Größe dem rechtsseitigen entspricht und deutlich Epididymis und Vas deferens erkennen läßt. Das Vas deferens dieses Hodens verläuft, soweit zu verfolgen, völlig getrennt von dem des kleineren Hodengebildes. Bruchsack, Leistenkanal und der dem rechten an Größe entsprechende Hoden werden wic rechts versorgt, das kleinere Hodengebilde samt seinem Funiculus entfernt und zur histologischen Untersuchung an dic Prosektur des Schwabinger Krankenhauses gesandt.

Der weitere klinische Verlauf des Falles war ein glatter. Der kleine Patient verließ am 31. VII. geheilt die Anstalt. Nach I Jahr war der Operationserfolg ein guter.

Der pathologisch-anatomische Befund (Prof. Oberndorfer) ist folgender: Makroskopis ch: Häutiges Gebilde, das mit seinen Anhängen gut einen erbsengroßen weichen Hoden mit entsprechend verkleinerten Gefäßen darstellen könnte. Mikroskopisch: Wie angenommen, handelt es sich um Überhoden, der hochgradig hypoplastisch ist, dessen Kanälchenepithel von hohen zylindrischen Zellen 
gebildet wird, die mit blasig aufgetriebenen, basalen Zellen untermengt sind. Die Hodenkanälchen sind von korbartigem, lockerem Bindegewebe umgeben, vereinzelt finden sich $Z$ wischenzellen in dem lockeren Gewebe. Ein Nebenhoden ist ausgebildet mit weiteren von Zylinderepithel ausgekleideten Hohlräumen und stärkerer Muskelwand der Kanäle. Ebenso ist ein kleines Vas deferens ausgebildet mit etwas niederem Zylinderepithel. Die Details sind aus dem anliegenden Lichtbild deutlich erkennbar.

Es hat sich also um einen linksseitigen, hypoplastischen dritten Hoden gehandelt, der im Gegensatz zu dem besser entwickelten, ebenfalls im Descensus zurückgebliebenen anderen linken Hoden

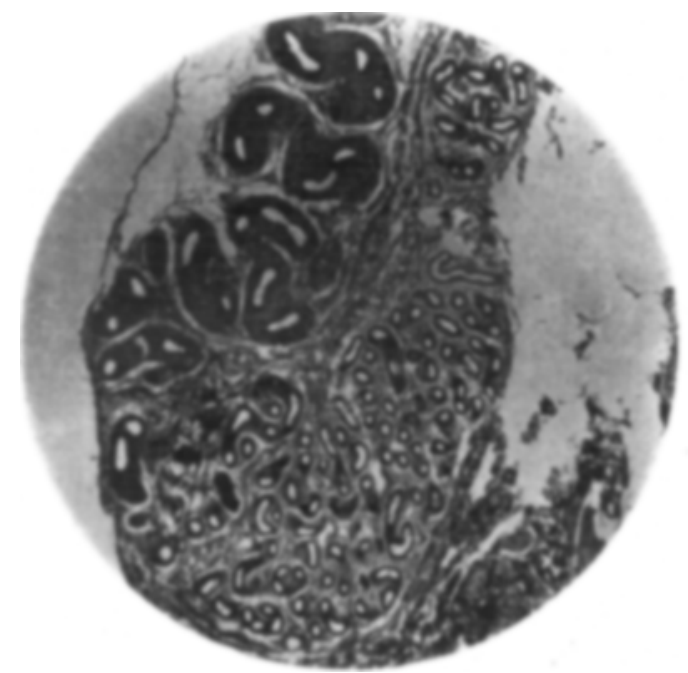

Fig. I.

nicht in dem kongenitalen Bruchsack lag. Der Hoden weist sowohl einen Nebenhoden wie einen echten Ductus deferens auf. Der Fall Lossens lag insofern anders, als sich bei ihm der scheinbare Ductus deferens des oberen Hodens mikroskopisch als aus Nebenhodenkanälchen bestehend erwies. Über den genauen histologischen Befund im Fall Mariottis gibt das Referat keine Auskunft.

Mit Ausnahme der Fälle Le Dent u und Lossen betreffen sämtliche Fälle die linke Seite.

Interessant sind die Variationen, die bei der Doppelbildung des Hodens bisher beobachtet sind. 
Hoden Nebenhoden Duct. defer.

Le Dentu

Lossen

2

Defranceschi

$\begin{array}{ll}2 & 2 \\ 1 & 1 \\ ? & 2\end{array}$

(2 durch Nebenhodenkanälchen verbundene Hälften)

$\mathrm{H}$ a a s

Widhalm

2

2

2

I
2

1

2

Der Fall Wid ha Im bietet Ähnlichkeit mit dem Fall Los se n. In beiden Fällen bestand nur ein Nebenhoden für beide Hoden, bei Lossen allerdings in zwei Hälften, die durch ausgezogene Nebenhodenkanälchen verbunden waren. Im Fall Defrance$\mathrm{schi}$ ist ohne histologische Untersuchung nicht mehr zu entscheiden, ob der in den Ductus des Haupthodens führende Strang ein echtes Vas deferens war.

Ich habe bei der großen Seltenheit der Anomalie die Veröffentlichung des Falles und die Zusammenstellung der bisherigen einschlägigen Literatur für gerechtfertigt gehalten. Es ist dadurch erwiesen, daß echte Hyperorchidie ohne Hermaphroditismus vorkommt, wenn sie auch als außerordentlich selten betrachtet werden muß.

\section{Literaturverzeictinis.}

I. A y yer, A triorchid. Lanc. 192I, Bd. 200, Nr. 5.

2. Defranceschi, Über einen Fall von 'Triorchismus. Bruns' Beitr. I9I0, Bd. 67 .

3. Drenkhahn, 3 Hoden. Deutsche Militärärztl. Zeitschr. 1912, Nr. 2.

4. Ko cher. Deutsche Chirurgie 1887 .

5. K ö n ig. Lehrbuch der speziellen Chirurgie, Bd. 2 ,

6. Le Dentu. Anomalies du testicule 1869 .

7. Lossen, Überzählige Hoden. Festschrift des Stadtkrankenhauses Dres. den-Friedrichstadt 1899 .

8. Mariotti, Un raro caso di Triorchismo. Gaz. degli ospedali e delle clin. 1907, Bd. 2. Nach Ref. im Zentralbl. f. Chir. 1907.

9. Potarca, Un cas authentique de Triorchidie. Semaine méd. 1907, 19.

ı. Widhalm, Ein Fall von linksseitigem Doppelhoden. Wiener med. Wochenschr. I9II, Nr.23. 